\title{
Monitoramento da hidrólise/condensação do tetraetoxisilano em solução com baixo teor alcoólico por análises de espectroscopia de infravermelho
}

\section{Monitoring tetraethoxysilane hydrolysis/condensation in solutions with low alcoholic content by infrared spectroscopy}

\author{
${ }^{1}$ Laboratório NanoTec - Departamento de Engenharia Civil (ECV), Universidade Federal de Santa Catarina (UFSC), \\ 88040-900, Florianópolis, Santa Catarina, Brasil. \\ e-mail: cezar.acasa@gmail.com, wellington.repette@gmail.com
}

\begin{abstract}
RESUMO
O objetivo desse trabalho foi monitorar a hidrólise/condensação do tetraetoxissilano (TEOS) em função do tempo de diluição do silano, por meio da análise de espectroscopia de infravermelho (IR). Inicialmente foram avaliadas duas concentrações de silano ( 1 e $10 \%$ em volume). No entanto, verificou-se que em concentrações de $1 \%$ de TEOS não foi possível identificar as ligações químicas de interesse ao monitoramento da hidrólise de maneira adequada em regiões características do espectro de infravermelho. Definida a concentração adequada, o monitoramento da hidrólise/condensação foi realizado por meio dos espectros de infravermelho obtidos em soluções hidrolisadas à temperatura ambiente e extraídas a cada 10 minutos. Verificou-se que as reações de hidrólise dos grupos alcóxi aceleraram em 40 minutos a partir da diluição do TEOS. Em paralelo, a condensação dos silanóis também acelerou, devido ao aumento na sua concentração na solução. Após 70 minutos, a hidrólise/condensação apresentou uma desaceleração por meio da formação de siloxano e devido à hidrólise completa dos grupos alcóxi do TEOS. A hidrólise do TEOS em soluções com baixo teor alcoólico (em torno de $50 \%$ em volume) não mostrou prejuízos para o monitoramento das reações de interesse do sistema, apresentando cinética de reação similar ao reportado na bibliografia, mostrando-se como alternativa aos sistemas com consumo excessivo de solventes orgânicos.
\end{abstract}

Palavras-chave: TEOS, hidrólise, espectroscopia de infravermelho, silano.

\section{ABSTRACT}

The objective of this work was to monitor the hydrolysis/condensation of tetraethoxysilane (TEOS) as a function of the silane dilution time, through infrared (IR) spectroscopy. Initially two concentrations of silane ( 1 and $10 \%$ by volume) were evaluated. However, it was found that at $1 \%$ of TEOS concentrations it was not possible to identify the chemical bonds of interest for the hydrolysis monitoring adequately in regions characteristic of the infrared spectrum. Once the appropriate concentration was defined, the hydrolysis/condensation monitoring was carried out using the infrared spectra obtained in solutions hydrolyzed at room temperature and extracted every 10 minutes. It was found that the hydrolysis reactions of the alkoxy groups accelerated in 40 minutes from the TEOS dilution. In parallel, the condensation of the silanols also accelerated, due to the increase in their concentration in the solution. After 70 minutes, the hydrolysis/condensation showed a deceleration through siloxane formation and due to the complete hydrolysis of the TEOS alkoxy groups. The hydrolysis of TEOS in solutions with low alcohol content (around $50 \%$ by volume) did not show any damage to the monitoring of the reactions of interest of the system, presenting reaction kinetics similar to those reported in the literature, showing up as an alternative to systems with excessive consumption of organic solvents.

Keywords: TEOS, hydrolysis, infrared spectroscopy, silane. 


\section{INTRODUÇÃO}

Estudado extensivamente a partir da segunda metade do século XX, o processo sol-gel baseia-se na formação de redes inorgânicas a partir de soluções com alcoxidos metálicos [1,2]. Por meio desse processo, inúmeros materiais podem, modificados [3] ou sintetizados como, por exemplo, na formação de partículas [4], na deposição de filmes [5,6] e formação de aerogéis [7].

O processo sol-gel é definido por se constituir de uma fase sol, uma fase gel e inúmeros processos que envolvem a transformação de sol para gel. A fase sol é uma dispersão coloidal em uma fase líquida, enquanto que e a fase gel é constituída de uma rede contínua envolta em um fluido. As reações sol-gel promovem o crescimento coloidal das partículas (sol) e a subsequente formação da rede contínua (gel) por meio de relações de hidrólise e condensação de precursores alcoxidos metálicos, comumente à base de silício [8].

Os alcoxissilanos são compostos químicos com moléculas que se baseiam no silício como elemento principal e que, na maioria das moléculas, apresentam dois tipos de interação química, presentes em suas extremidades, sendo uma inorgânica e outra orgânica. São denominados, de forma geral, por um grupo hidrolisável do tipo alcóxi e um grupo organofuncional (amina $\left(-\mathrm{NH}_{2}\right)$, vinil $(-\mathrm{C}=\mathrm{C})$ ). $\mathrm{O}$ grupo alcóxi pode ocupar a posição do grupo organofuncional, tal como o tetraetoxissilano (TEOS) e o tetrametoxissilano (TMOS). Dentre os silanos, o TEOS é o mais utilizado [2,9].

O silano é normalmente encontrado no estado não hidrolisado e, para reagir, primeiramente necessita ser hidrolisado para gerar grupos silanóis $(\equiv \mathrm{Si}-\mathrm{OH})$, que são responsáveis pela reação do silano com um substrato ou ainda possibilitam a formação da fase gel [10].

Os fenômenos de hidrólise e de condensação são considerados de alta complexidade, pois ocorrem simultaneamente, e apresentam taxas de reações variáveis ao longo do processo [11]. Alguns parâmetros interferem diretamente nas velocidades de hidrólise e de condensação dos silanos, tais como: o tipo do grupo organofuncional (aniônico ou catiônico), o pH da solução, a concentração da solução (silano: solvente alcoólico), o envelhecimento da solução e a temperatura [2,12]. No entanto, o pH é o que mais afeta a velocidade de hidrólise/condensação dos silanos, pois ambas reações são catalisadas por bases ou ácidos e proporcionando alterações do $\mathrm{pH}$ ao longo das reações de hidrólise $[8,13]$.

Nos processos realizados com TEOS e outros alcoxissilanos, não há consenso sobre qual a duração mais adequada para o processo da hidrólise dos silanos. Isto é, por ser altamente afetado pelos fatores intervenientes na reação [14]. Em aplicações específicas, como é a deposição de filmes finos em superfícies metálicas por dip-coating ou por aspersão, um dos fatores mais importantes para garantir a uniformidade e a integridade da cobertura é a duração do processo de hidrólise dos silanos, que é definido pelo intervalo de tempo desde a diluição do silano até a utilização do silano hidrolisado. Isso se dá pelo fato de haver um período após a diluição do silano em que há maior concentração de grupos silanóis reativos em solução, para a aplicação do filme à superfícies [12].

Devido à baixa miscibilidade dos alcoxissilanos em água, a hidrólise das moléculas de silanos necessita da presença de um solvente orgânico para a "compatibilização" e composição da solução de hidrólise. Comumente, o solvente orgânico mais empregado é um álcool [15] e, nos processos sol-gel envolvendo silanos empregam-se solventes com concentrações de álcool superiores à 80\% em volume [16].

A longa duração do processo de hidrólise e alto consumo de solventes alcoólicos resultam em processos ambientalmente pouco corretos. Pois, faz uso de grandes quantidades de compostos orgânicos voláteis (VOC's) e normalmente são poucas as bibliografias que reportam estudos que visam a diminuição no processo sol-gel.

Este trabalho, tem como objetivo investigar e monitorar, através de espectroscopia de infravermelho, a cinética de reação da hidrólise/condensação do silano TEOS, catalisadas em meio ácido, em função do tempo de diluição do silano.

\section{PROGRAMA EXPERIMENTAL}

\subsection{Materiais}

Para o processo foi utilizado o TEOS (Tetraetoxissilano), cujas propriedades estão apresentadas na Tabela 1. Utilizou-se ácido acético glacial $\left(\mathrm{CH}_{3} \mathrm{COOH}\right)$ com concentração superior aos $99 \%$, como regulador de $\mathrm{pH}$, produzido pela ALDRICH (EUA). Como solvente alcoólico, empregou-se etanol $\left(\mathrm{C}_{2} \mathrm{H}_{6} \mathrm{O}\right)$ com concentração superior aos 96\% da ALDRICH (EUA). 
Tabela 1: Características do TEOS

\begin{tabular}{l|l}
\hline NOME & TETRAETOXISSILANO \\
\hline Sigla & TEOS \\
\hline Fabricante & Aldrich (EUA) \\
\hline Pureza & $>98 \%$ \\
\hline Grupo hidrolisável & Etóxi \\
\hline Grupo funcional & - \\
\hline Fórmula química & $\mathrm{C}_{8} \mathrm{H}_{20} \mathrm{O}_{4} \mathrm{Si}$ \\
\hline Massa molar (g/mol) & 208,33 \\
\hline & $\mathrm{H}_{3} \mathrm{C}$ \\
Fórmula estrutural & \multicolumn{2}{|c}{$\mathrm{CH}_{3} \mathrm{C}$} \\
\hline
\end{tabular}

\subsection{Promoção da hidrólise/condensação e monitoramento por espectrometria de infravermelho}

Durante a caracterização das amostras por infravermelho, utilizou-se um espectrômetro Jasco modelo FT/IR4200, com um acessório de refletância total atenuada - ATR PRO450-S/470-H, com cristal de seleneto de zinco (ZnSe) (Figura 1). Para cada espectro analisado, foram realizados 42 scans no intervalo de número de onda de 4000 a $550 \mathrm{~cm}^{-1}$ e resolução de $0,5 \mathrm{~cm}^{-1}$. Para a análise das soluções, depositaram-se gotas da amostra no compartimento porta amostra, de modo a cobrir todo o cristal do ATR. Todos os espectros foram avaliados com os programas Spectral analysis, da Jasco, e Knowitall ${ }^{\circledR}$, da Bio-Rad Laboratories.

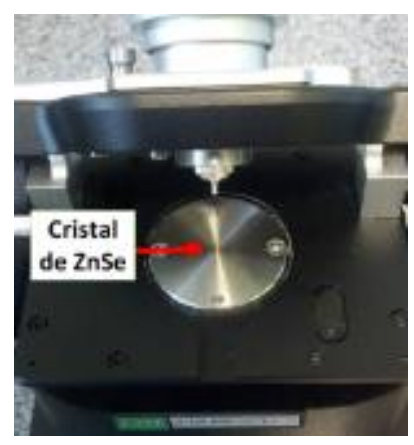

(a)

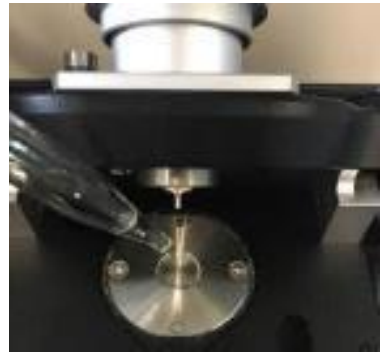

(b)

Figura 1: Equipamento para análise de infravermelho. (a) Compartimento da amostra no acessório ATR; (b) Deposição da amostra líquida cobrindo o cristal do ATR.

Para a diluição do TEOS, utilizou-se uma solução alcoólica formada por etanol e água destilada na proporção de 1: 1 (em volume), acidificado com ácido acético até a estabilização do pH em $5 \pm 0,2$ (chamado de solvente alcoólico). Para a análise da hidrólise do TEOS, foi realizada a caracterização dos reagentes químicos e do solvente alcoólico acidificado por meio de infravermelho.

Em geral, os experimentos envolvendo soluções de silanos diluídos são realizados e recomendados com concentrações entre $1 \%$ e $10 \%$ em volume. Assim, para verificar se com o equipamento utilizado seria possível o monitoramento da hidrólise do TEOS na solução de concentrações na ordem de $1 \%$, realizou-se análise comparativa dos espectros de infravermelho do TEOS concentrado e de diluições de $1 \%$ e $10 \%$ (em volume), conforme Figura 2 (a). A extração de alíquotas para a realização da análise nas amostras diluídas foi realizada 25 segundos após a diluição do TEOS.

Depois de determinada a concentração adequada para o monitoramento da hidrólise, realizou-se a 
análise do monitoramento da hidrólise em função do tempo de diluição do TEOS. O método consistiu em formular uma solução com 90\%(vol.) de solução alcoólica acidificada e 10\%(vol.) de TEOS.

A mistura foi mantida em agitação constante sob temperatura ambiente $\left(25 \pm 2{ }^{\circ} \mathrm{C}\right)$. Realizou-se a partir da diluição do TEOS, extrações de alíquotas de aproximadamente 0,5 ml da mistura a cada 10 minutos, por um período total de 120 minutos. Dentre a coleta das amostras até a determinação do espectro de infravermelho, levou aproximadamente 40 segundos. Os procedimentos descritos estão ilustrados na Figura 2 (b).

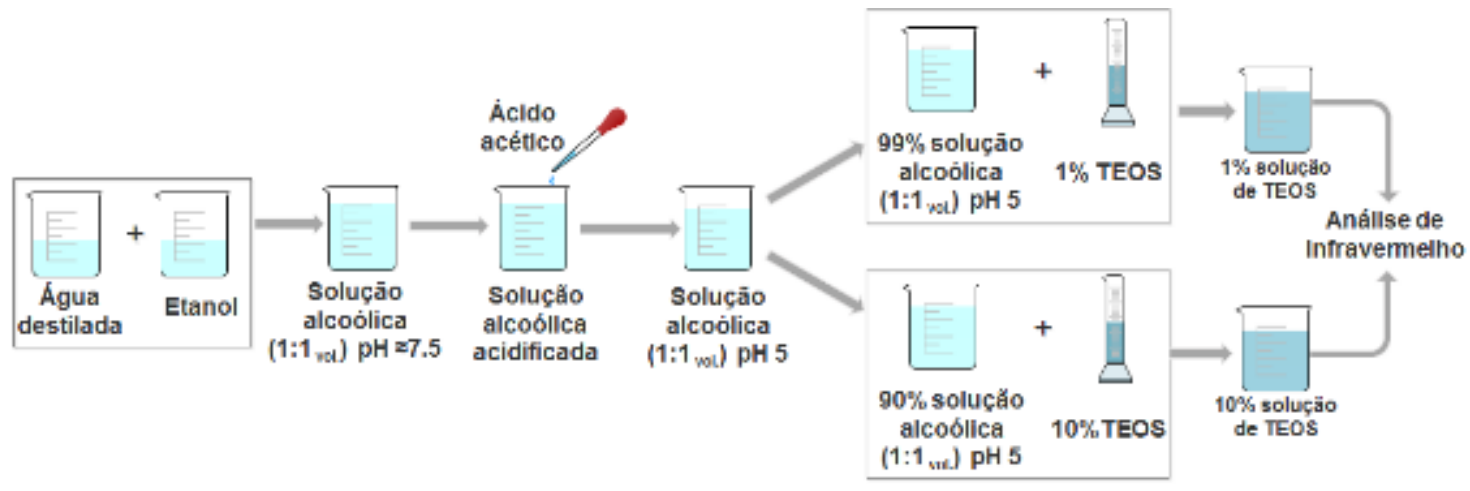

(a)

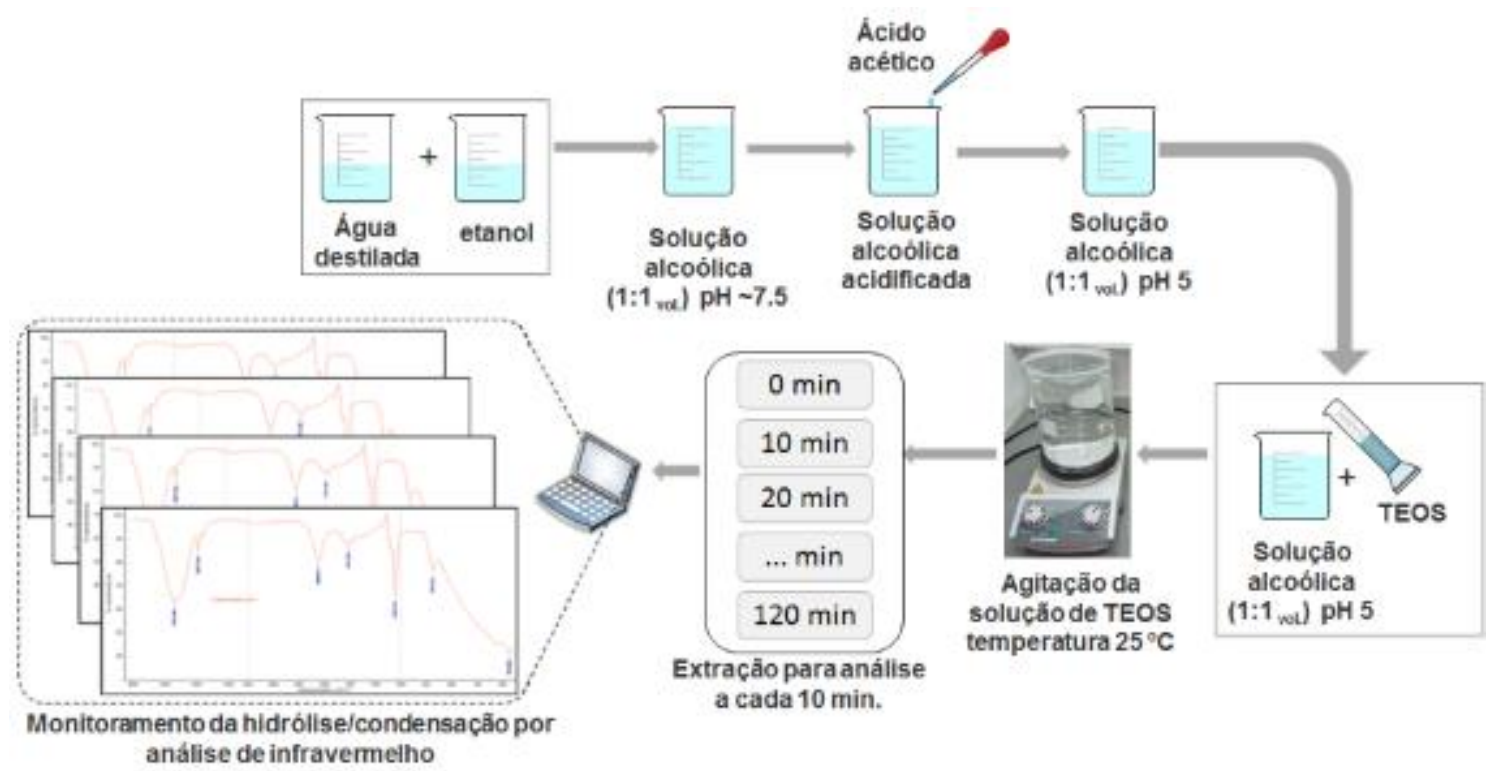

(b)

Figura 2: Método de extração e análise das amostras por infravermelho. (a) Análise da concentração de TEOS na influência da detecção do espectro; (b) Análise do monitoramento da hidrólise do TEOS em função do tempo.

\section{RESULTADOS E DISCUSSÕES}

A sessão de resultados será apresentada em duas etapas. Na primeira, serão apresentados os resultados da análise da sensibilidade do equipamento e as caracterizações dos componentes e dos produtos empregados, bem como a definição de parâmetros para a etapa seguinte. Na segunda, serão mostrados os resultados do estudo do monitoramento da hidrólise do TEOS em função do tempo. 


\subsection{Análise da sensibilidade do equipamento e parâmetros de ensaio}

A Figura 3 exibe os espectros de infravermelho dos reagentes empregados na hidrólise do TEOS e, na Tabela 2, as respectivas ligações químicas correspondentes às bandas identificadas.

Verificou-se, na água, picos intensos nas regiões do espectro dos $3350 \mathrm{~cm}^{-1}, 1640 \mathrm{~cm}^{-1}$ e com um pico largo e progressivo a partir dos $1000 \mathrm{~cm}^{-1}$ aos $550 \mathrm{~cm}^{-1}$ sendo característico na região dos $580-550 \mathrm{~cm}^{-1}$.

No ácido acético verificou-se várias regiões de bandas características, como a do grupo acetato na região dos 2950-2900 $\mathrm{cm}^{-1}$ e $1430-1380 \mathrm{~cm}^{-1}$ referente a ligação O-H. Na região de $1725-1700 \mathrm{~cm}^{-1}$ foi verificado a banda referente ao grupo $\mathrm{C}=\mathrm{O}$ e na região de $1114 \mathrm{~cm}^{-1}$ referente a ligação $\mathrm{C}-\mathrm{O}$.

No espectro do etanol foi verificado bandas na região dos $3400-3200 \mathrm{~cm}^{-1}$, dos $1430-1380 \mathrm{~cm}^{-1}$ e 879 $\mathrm{cm}^{-1}$ referente à ligação O-H. A ligação característica de C-H foi detectada na região dos $2970 \mathrm{~cm}^{-1}$ referente à cadeia carbônica, e na região dos 1080-1045 cm $\mathrm{cm}^{-1}$ referente à ligação C-O.

No TEOS, foram identificadas nas regiões dos 2978-2900 cm-1 e 2950-2900 cm aos grupos $\mathrm{C}-\mathrm{H}_{2}$ e C- $\mathrm{H}_{3}$, respectivamente. A banda referente às ligações de silanol ( $\left.\mathrm{Si}-\mathrm{OH}\right)$ foram detectadas na região dos 960-900 $\mathrm{cm}^{-1}$. No caso da identificação das ligações de siloxano (Si-O-Si) verificou-se que há uma sobreposição de bandas, sendo a banda referente a ligação Si-O-C identificada na mesma região de comprimento de onda, na faixa dos $1160 \mathrm{~cm}^{-1}$ e $1080-1070 \mathrm{~cm}^{-1}$.

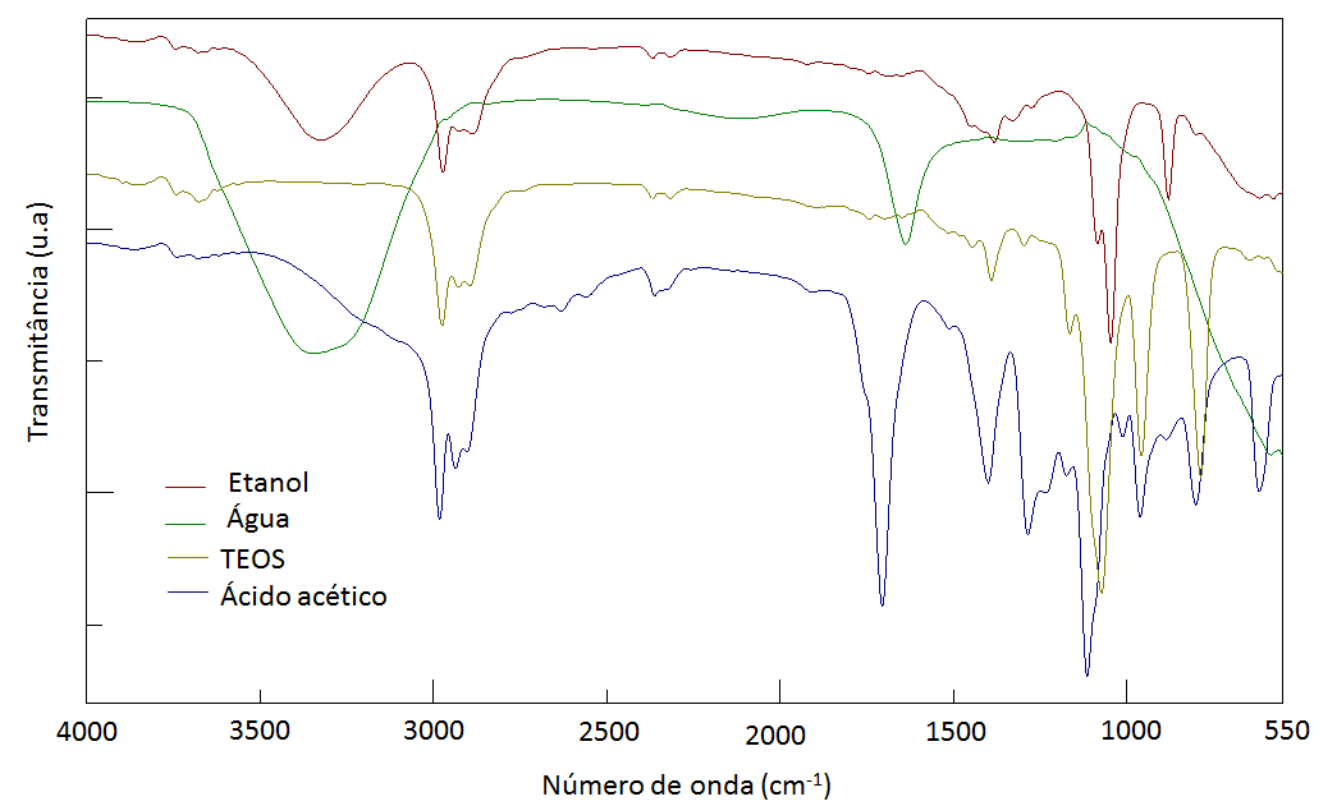

Figura 3: Espectros infravermelho dos reagentes utilizados na hidrólise. 
Tabela 2: Bandas características dos reagentes utilizados na hidrólise $[4,9,16-23]$

\begin{tabular}{|c|c|c|c|c|}
\hline NÚMERO DE ONDA $\left(\mathrm{cm}^{-1}\right)$ & ÁGUA & ETANOL & TEOS & ÁCIDO ACÉTICO \\
\hline $3400-3200$ & ${ }_{v} \mathrm{O}-\mathrm{H}$ & ${ }_{v} \mathrm{O}-\mathrm{H}$ & & \\
\hline $2978-2970$ & & ${ }_{v s} \mathrm{C}-\mathrm{H}_{2}$ & ${ }_{v s} \mathrm{C}-\mathrm{H}_{2}$ & ${ }_{v s} \mathrm{C}-\mathrm{H}$ \\
\hline $2950-2900$ & & & vas $\mathrm{C}-\mathrm{H}_{3}$ & vas $\mathrm{C}-\mathrm{H}_{3} / \mathrm{O}-\mathrm{H}$ \\
\hline $1725-1700$ & & & & ${ }_{a} \mathrm{C}=\mathrm{O}$ \\
\hline$\cong 1640$ & ${ }_{\delta} \mathrm{O}-\mathrm{H}$ & & & \\
\hline $1430-1380$ & & ${ }_{\delta} \mathrm{O}-\mathrm{H}$ & ${ }_{\delta a s} \mathrm{C}-\mathrm{H}$ & $\delta_{\delta} \mathrm{O}-\mathrm{H}$ \\
\hline$\cong 1284$ & & & & $\delta a s \mathrm{C}-\mathrm{O}$ \\
\hline$\cong 1160$ & & & vas $\mathrm{Si}-\mathrm{O}-\mathrm{C}$, vas $\mathrm{Si}-\mathrm{O}-\mathrm{Si}$ & \\
\hline$\cong 1114$ & & & & ${ }_{\nu} \mathrm{C}-\mathrm{O}$ \\
\hline $1080-1070$ & & ${ }_{v} \mathrm{C}-\mathrm{O}$ & vas $\mathrm{Si}-\mathrm{O}-\mathrm{C}$, vas $\mathrm{Si}-\mathrm{O}-\mathrm{Si}$ & \\
\hline$\cong 1045$ & & ${ }_{v} \mathrm{C}-\mathrm{O}$ & & \\
\hline $960-900$ & & & ${ }_{\mathrm{v} \beta} \mathrm{Si}-\mathrm{O}(\mathrm{H})$ & ${ }_{v s} \mathrm{C}-\mathrm{H}$ \\
\hline$\cong 879$ & & ${ }_{v} \mathrm{O}-\mathrm{H}$ & & \\
\hline $802-780$ & & & ${ }_{v s} \mathrm{Si}-\mathrm{O}$ & ${ }_{\nu} \mathrm{C}-\mathrm{H}$ \\
\hline$\cong 617$ & ${ }_{o p} \mathrm{O}-\mathrm{H}$ & & & ${ }_{\nu} \mathrm{C}-\mathrm{H}$ \\
\hline $580-550$ & $v \mathrm{O}-\mathrm{H}$ & & & \\
\hline
\end{tabular}

Obs.: v: vibração de alongamento; vs: vibração de alongamento simétrica; vas: vibração antissimétrica; $\delta$ : vibração de flexão; $\delta a s$ : vibração de flexão antissimétrica; $v \beta$ : vibração de alongamento no plano; $a$ : vibração de deformação axial; op: vibração de deformação fora do plano.

Verificou-se que as bandas no espectro da água são intensas, cobrindo grande parte do espectro na região de comprimento de onda 4000-550 $\mathrm{cm}^{-1}$, o que pode dificultar a análise devido ao volume de água na solução (cerca de 50\%) ser expressivo e sobrepor as ligações químicas de interesse das reações de hidrólise do TEOS. Outro fator é o impacto do ácido acético na mistura, pois a sua presença se resume a algumas gotas no ajuste do $\mathrm{pH}$ de 7 para 5. Isto é, apesar de estar presente, o ácido acético não afetou o espectro da solução utilizada como solvente.

No caso de silanos, há bandas importantes que podem se sobrepor, como na região de $1160 \mathrm{~cm}^{-1}$, indicando a ligação de siloxanos ( $\equiv \mathrm{Si}-\mathrm{O}-\mathrm{Si} \equiv)$ e alcóxi ( $\equiv \mathrm{Si}-\mathrm{O}-\mathrm{C})$, sendo essa região de complexa na análise. Também pode haver sobreposição de bandas na região de $960-900 \mathrm{~cm}^{-1}$, onde as bandas referentes à

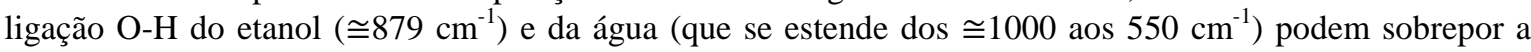
ligação referente ao silanol ( $\equiv \mathrm{Si}-\mathrm{OH})$.

Assim, realizou-se uma análise comparativa dos espectros das soluções alcoólicas acidificadas com os silanos em seu estado puro, para verificar se seria possível identificar as bandas de maior interesse referentes a hidrólise/condensação, que são nos comprimentos de onda da região de 1160, 1080-1070, 960-900 e 802$780 \mathrm{~cm}^{-1}$, apresentado na Figura 4. As bandas do TEOS, na região de 1160, 1080-1070, 960-900 e 802-780 $\mathrm{cm}^{-1}$ não são sobrepostas pelas bandas características da solução alcoólica. Apesar da possibilidade de identificação de bandas características da hidrólise/condensação, devido as soluções serem compostas de quantidades expressivas de solvente (superior a 90\%), realizou-se a diluição, de 1 e $10 \%$ de TEOS na solução alcoólica acidificada afim de verificar se seria possível a identificação das bandas de interesse. 


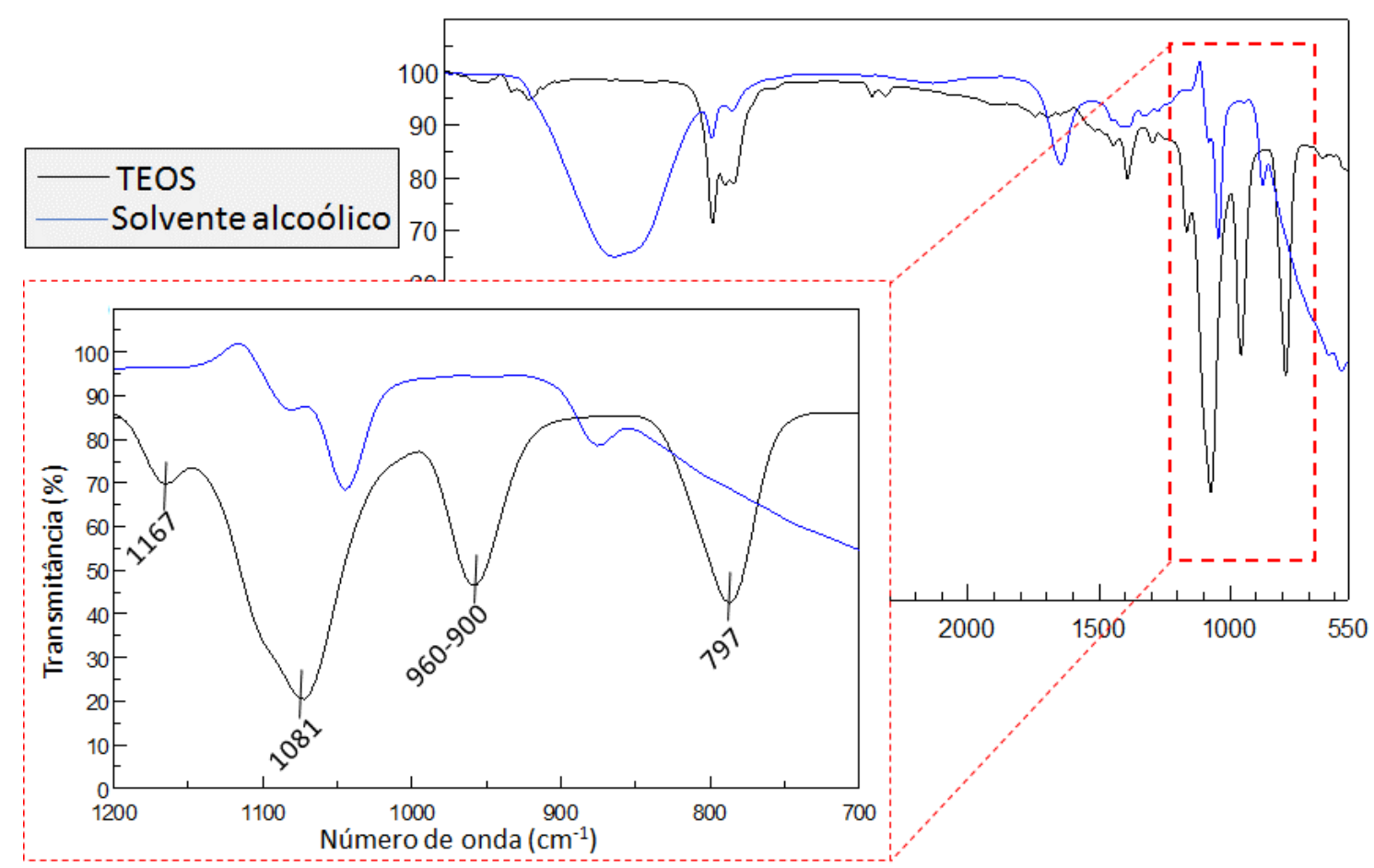

Figura 4: Análise comparativa dos espectros do TEOS concentrado e da solução alcoólica para verificação das bandas de interesse

Na Figura 5 são apresentados os espectros para o TEOS e os espectros das soluções com $1 \%$ e $10 \%$ de TEOS. São mostrados os espectros no intervalo de comprimento de onda de $4000-550 \mathrm{~cm}^{-1}$ e há um detalhe em $1200-700 \mathrm{~cm}^{-1}$.

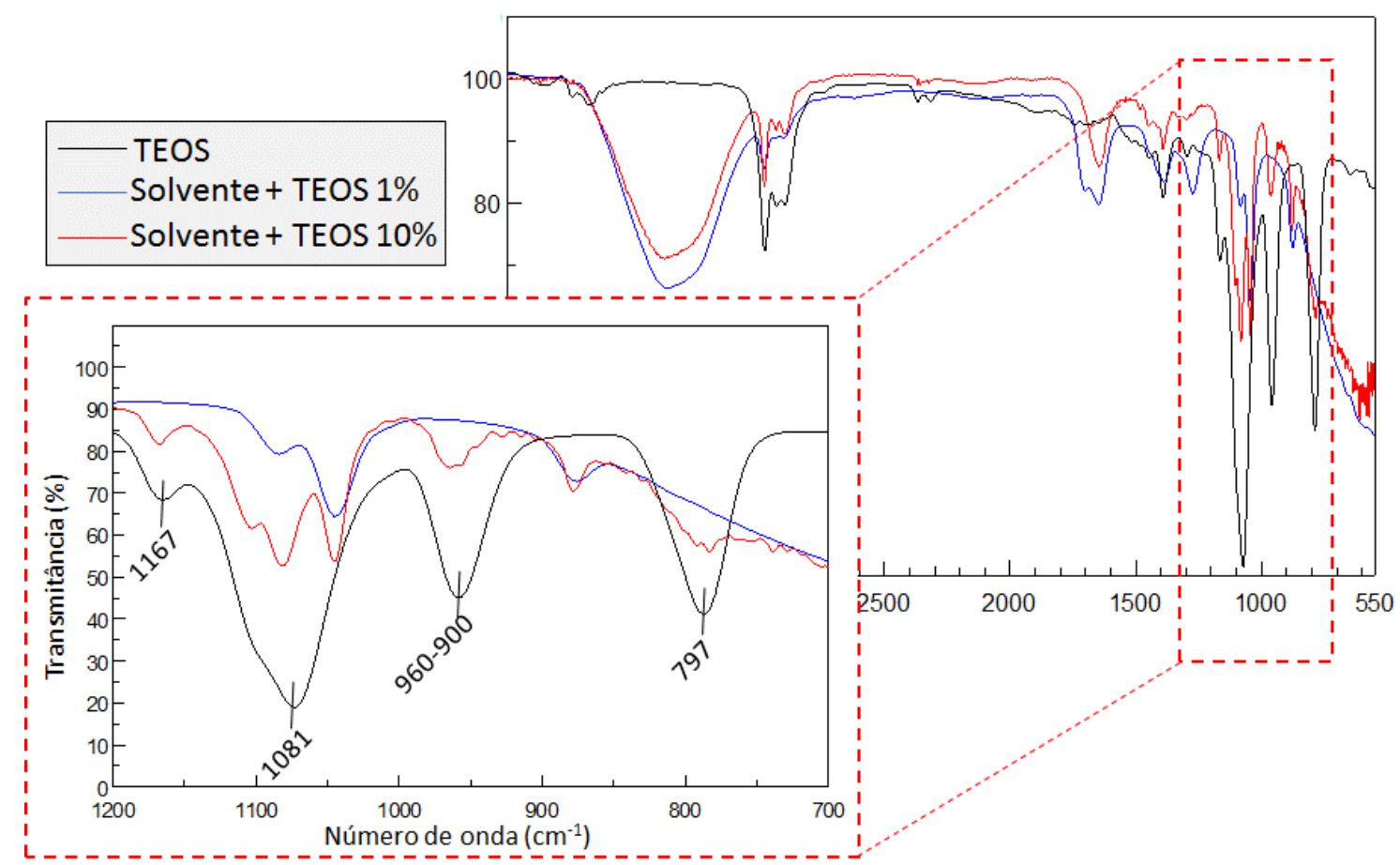

Figura 5: Análise comparativa dos espectros do TEOS concentrado e das soluções alcoólicas com concentrações de TEOS de 1 e $10 \%$ 
Para as duas concentrações investigadas, somente na solução com concentração de $10 \%$ foi possível identificar as bandas de interesse para o monitoramento da hidrólise/condensação do TEOS. Na região dos $1167 \mathrm{~cm}^{-1}$, o pico foi detectado somente para a concentração de $10 \%$. Na região dos $1080-1070 \mathrm{~cm}^{-1}$ foram identificados picos nas duas concentrações estudadas, porém essa região é complicada de identificar, além de compartilhar a banda da ligação referente ao C-O do etanol que está presente na solução na ordem de $50 \%$ em volume. O pico na região dos $960-900 \mathrm{~cm}^{-1}$, referente à ligação do silanol, só foi detectado na solução com a concentração de $10 \%$ de silano. Além disso, não foi possível identificar a banda na região dos 802-780 $\mathrm{cm}^{-1}$ com de $1 \%$ de TEOS na solução, pois o espectro do solvente alcoólico sobrepôs toda a região do espectro, já na solução com concentração de $10 \%$ houve a possibilidade de identificação dessa banda.

Assim, não houve possibilidade de realizar uma análise adequada sobre a hidrólise/condensação do TEOS com na solução com $1 \%$ de concentração. Não foi possível identificar as bandas de interesse, pelo fato de estarem sobrepostas pelo espectro da própria solução alcoólica, o que pode ser uma limitação da própria configuração da solução utilizada ou da sensibilidade do equipamento utilizado. A partir desses resultados, o monitoramento da hidrólise/condensação do TEOS foi realizado com a solução de concentração de $10 \%$ em volume de silano.

\subsection{Monitoramento da hidrólise do TEOS}

Na Figura 6 são apresentados os espectros obtidos a partir da diluição de 10\% de TEOS no solvente alcoólico com intervalos entre as análises de 10 minutos, no comprimento de onda de $4000-550 \mathrm{~cm}^{-1}$ e ampliação do espectro em 1200-700 $\mathrm{cm}^{-1}$. O tempo de hidrólise indicado na parte superior do espectro é referente ao momento da coleta desde o momento de diluição do TEOS na solução. As barras verticais azuis indicam os picos referentes às ligações químicas de interesse para o monitoramento da hidrólise/condensação nos comprimentos de onda de $1160,1080-1070,960-900$ e $802-780 \mathrm{~cm}^{-1} . \mathrm{Na}$

Tabela 3 são apresentados os valores quantitativos das intensidades de transmitância das bandas selecionadas, determinados a cada 10 minutos. 

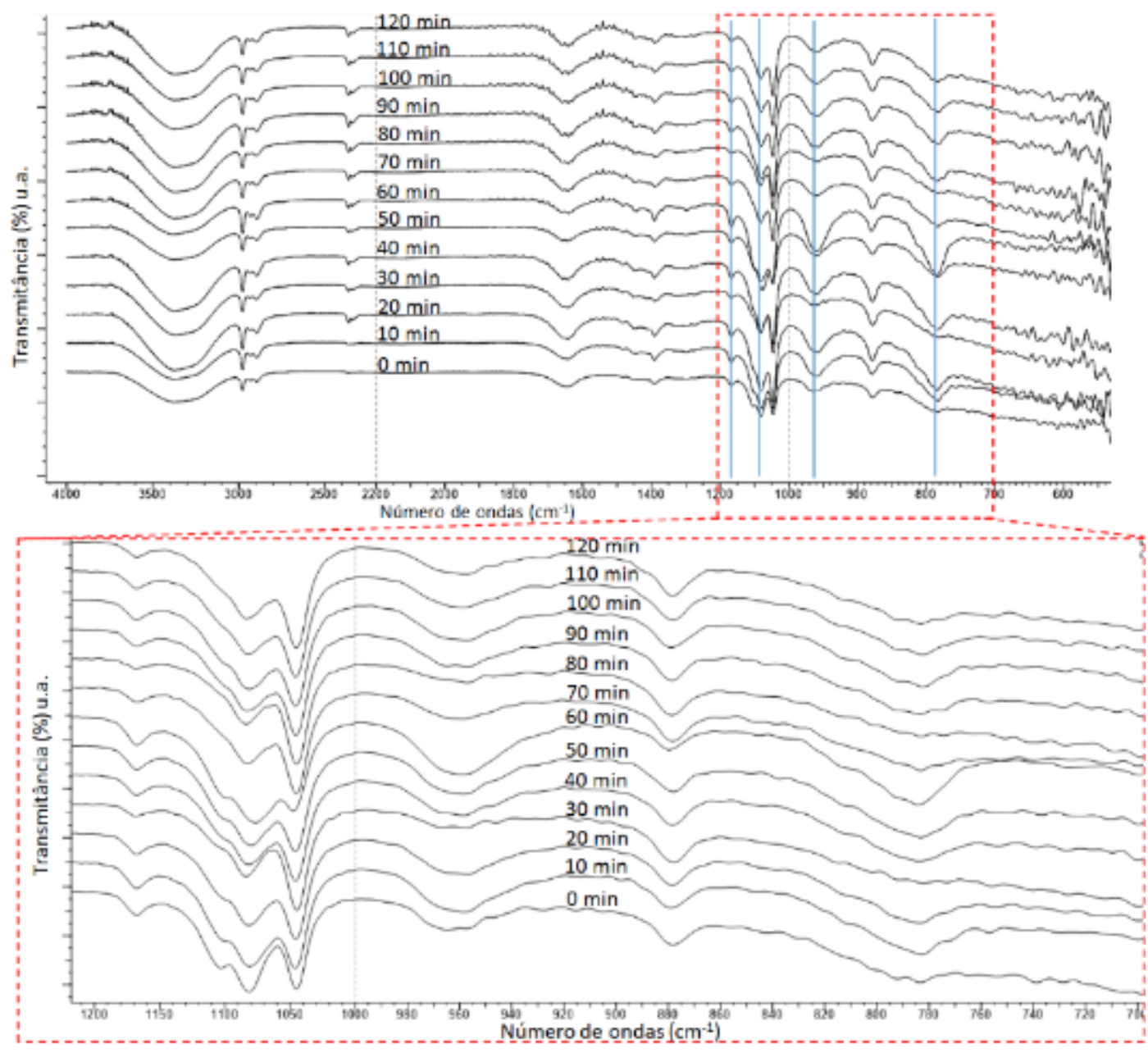

Figura 6: Espectros de infravermelho do TEOS para o monitoramento da hidrólise em função do tempo. Espectros nos limites 4000-550 $\mathrm{cm}^{-1}$; barras verticais azuis indicando os comprimentos de onda de 1160, 1080-1070, 960-900 e 802-780 $\mathrm{cm}^{-1}$; ampliação em 1200-700 $\mathrm{cm}^{-1}$.

Tabela 3: Transmitância das bandas características da hidrólise do TEOS

\begin{tabular}{l|l|l|l|l}
\hline \multirow{2}{*}{ TEMPO (MINUTOS) } & \multicolumn{4}{|c}{ TRANSMITÂNCIA (\%) } \\
\cline { 2 - 5 } & $\mathbf{1 1 6 7 \mathbf { c m } ^ { - 1 }}$ & $\mathbf{1 0 8 1} \mathbf{c m}^{-1}$ & $\mathbf{9 6 0} \mathbf{~ m}^{-1}$ & $\mathbf{7 8 3} \mathbf{c m}^{-1}$ \\
\hline 0 & 92,10 & 66,90 & 84,10 & 65,60 \\
\hline 10 & 90,40 & 62,70 & 85,40 & 63,40 \\
\hline 20 & 91,20 & 63,50 & 83,60 & 64,30 \\
\hline 30 & 91,30 & 63,80 & 83,10 & 64,50 \\
\hline 40 & 90,00 & 63,70 & 82,80 & 65,00 \\
\hline 50 & 90,10 & 59,70 & 78,70 & 63,00 \\
\hline 60 & 86,50 & 49,20 & 73,10 & 58,10 \\
\hline 70 & 87,40 & 50,50 & 85,10 & 59,70 \\
\hline 80 & 88,40 & 51,50 & 85,30 & 60,10 \\
\hline 90 & 87,60 & 51,90 & 85,40 & 60,90 \\
\hline 100 & 88,10 & 49,90 & 84,30 & 60,10 \\
\hline 110 & 88,00 & 50,20 & 85,70 & 59,90 \\
\hline 120 & 87,50 & 49,80 & 85,50 & 60,40 \\
\hline
\end{tabular}


As alterações significativas para a hidrólise/condensação do TEOS ocorreram ao redor de 60 minutos de hidrólise (Figura 6). Isto é, os resultados evidenciaram um aumento expressivo na taxa de hidrólise ao redor deste período de tempo.

Na região do espectro referente aos grupos silanóis ( $\mathrm{Si}-\mathrm{OH}$ ), a banda na região de $960 \mathrm{~cm}^{-1}$ apresentou uma pequena diminuição da sua transmitância até o tempo de 40 minutos. Isto significa que, apesar de pouco evidente, já ocorreu a hidrólise dos grupos Si-O-C do silano logo nos primeiros momentos da sua diluição. Assim que o TEOS foi adicionado à solução, houve a formação de silanóis de natureza ácida, que tendem a acelerar a reação de hidrólise e, concomitantemente com a hidrólise, houve a formação de etanol, o que favorece a solubilidade do TEOS na solução. A reação não acontece simultaneamente para os grupos alcóxi [24], sendo hidrolisados intercaladamente, de modo que para cada mol de silanol gerado na solução, também forma-se um mol de etanol (como mostrado na Equação 1). No período de 40 a 60 minutos, ocorreu uma diminuição intensa nos valores de transmitância, indicando o aumento expressivo na taxa de hidrólise e o aumento da formação dos silanóis nesse período (como mostrado nas Equações 2 e 3). Após 50 minutos, ocorreu a hidrólise da maior parte das moléculas do silano (Equação 4), evidenciado pela diminuição progressiva da transmitância da banda de $960 \mathrm{~cm}^{-1}$ até os 60 minutos.

Os silanóis formados pela hidrólise do TEOS tendem a condensam com as moléculas vizinhas para a formação de moléculas oligoméricas, resultando na liberação de água que leva à diminuição da solubilidade do TEOS, impactando nas reações e causando a redução da taxa de hidrólise (Equação 5). Depois de 60 min, o valor de transmitância na região de $960 \mathrm{~cm}^{-1}$ retornou a valores próximos aos observados no início da hidrólise, decorrente da condensação dos grupos silanóis. O monitoramento da banda de $960 \mathrm{~cm}^{-1}$ evidenciou que no intervalo de tempo investigado, o período com maior índice de grupos silanóis na solução foi entre 50 e 60 minutos.

$$
\begin{array}{lr}
\mathrm{SiO}_{4}\left(\mathrm{C}_{2} \mathrm{H}_{5}\right)_{4}+\mathrm{H}_{2} \mathrm{O} \rightarrow \mathrm{HOSiO}_{3}\left(\mathrm{C}_{2} \mathrm{H}_{5}\right)_{3}+\mathrm{C}_{2} \mathrm{H}_{6} \mathrm{O} & 10-20 \mathrm{~min} \\
\mathrm{HOSiO}_{3}\left(\mathrm{C}_{2} \mathrm{H}_{5}\right)_{3}+\mathrm{H}_{2} \mathrm{O} \rightarrow(\mathrm{HO})_{2} \mathrm{SiO}_{2}\left(\mathrm{C}_{2} \mathrm{H}_{5}\right)_{2}+2 \mathrm{C}_{2} \mathrm{H}_{6} \mathrm{O} & 20-40 \mathrm{~min} \\
(\mathrm{HO})_{3} \mathrm{SiO}_{3}\left(\mathrm{C}_{2} \mathrm{H}_{5}\right)_{2}+\mathrm{H}_{2} \mathrm{O} \rightarrow(\mathrm{HO})_{3} \mathrm{SiO}\left(\mathrm{C}_{2} \mathrm{H}_{5}\right)+3 \mathrm{C}_{2} \mathrm{H}_{6} \mathrm{O} & 40-60 \mathrm{~min} \\
\mathrm{SiO}_{4}\left(\mathrm{C}_{2} \mathrm{H}_{5}\right)_{4}+4 \mathrm{H}_{2} \mathrm{O} \rightarrow \mathrm{Si}(\mathrm{OH})_{4}+4 \mathrm{C}_{2} \mathrm{H}_{6} \mathrm{O} & 40-60 \mathrm{~min} \\
\mathrm{Si}(\mathrm{OH})_{4}+\mathrm{Si}(\mathrm{OH})_{4} \leftrightarrow \mathrm{Si}_{2} \mathrm{O}(\mathrm{OH})_{6}+\mathrm{H}_{2} \mathrm{O} & >60 \mathrm{~min}
\end{array}
$$

Pela banda na região dos $1167 \mathrm{~cm}^{-1}$, verificou-se uma pequena diminuição da transmitância da banda caracterizando maior concentração de siloxano ao longo do tempo analisado. Em torno de 60 minutos de hidrólise/condensação, houve uma mudança mais significativa no aumento da taxa de policondensação dos silanóis, devido à maior concentração de silanóis nesse período de tempo. Logo após os 60 minutos não houve variação significativa na sua transmitância.

Nas bandas da região de $1081 \mathrm{~cm}^{-1}$ e $783 \mathrm{~cm}^{-1}$ o comportamento foi semelhante ao verificado na banda $1167 \mathrm{~cm}^{-1}$. Isto é, também houve a formação de siloxano logo nos períodos iniciais, na diluição do silano, e permaneceu sem variação significativa até em torno dos 40 minutos. A partir deste momento, as reações de policondensação aceleraram devido à maior concentração de silanol na solução, resultando na diminuição expressiva nas suas transmitâncias até em torno dos 60 minutos, comprovando que a condensação do silano é intensificada nesse período de tempo.

Segundo Télles et al. [9] como resultado da rápida condensação dos silanóis, formam-se, primeiramente, entre 25 e 30 minutos desde o início da hidrólise, as moléculas com ligações de siloxanos lineares. Depois dos 60 minutos, as reações de hidrólise são desaceleradas bruscamente. Isto se deve pela própria policondensação das moléculas do silano parcialmente hidrolisadas, dificultando o acesso das moléculas de água para a hidrólise dos grupos alcóxi restantes e pela formação de moléculas tridimensionais. Além disso, a taxa de hidrólise tende a ser cada vez menor, pelo fato dos grupos $\mathrm{Si}-\mathrm{OH}$ assim que formados, tenderem a condensar com os silanóis disponíveis. Apesar da desaceleração, verificou-se pela banda 1167 $\mathrm{cm}^{-1}$ que a condensação dos silanóis continua, porém em velocidades lentas, também verificado por Cunliffe et al. [25].

A partir da investigação realizada, houve diferentes intensidades de hidrólise/condensação do TEOS. 
Até os 40 minutos: a taxa de hidrólise/condensação foi pouco significativa, porém, já houve a formação de grupos Si-OH e Si-O-Si lineares a partir da hidrólise do TEOS. Em torno de 50 minutos: houve um aumento intenso na taxa de hidrólise dos grupos $\mathrm{Si}-\mathrm{O}-\mathrm{C}$ para a formação dos grupos $\mathrm{Si}-\mathrm{OH}$. Em paralelo, a condensação do silano para a formação dos grupos Si-O-Si também é acelerada neste período. Porém, exibe aumento na sua intensidade com 10 minutos de atraso. A partir dos 60 minutos, onde as reações de hidrólise/ policondensação são desaceleradas.

\section{CONCLUSÕES}

Em síntese, as principais conclusões foram:

Esse estudo evidenciou como a hidrólise do TEOS pode ser conduzida em soluções com baixo teor alcoólico (50\% em volume);

Para o monitoramento da hidrólise do TEOS em soluções alcoólicas por meio de análise de infravermelho, dentre 1 e $10 \%$ recomenda-se utilizar concentrações de silano de $10 \%$ em volume, por não ser possível a identificação das bandas características de interesse quando $1 \%$ de TEOS foi utilizado;

O processo mostrou três taxas características de hidrólise/condensação: da diluição até os 40 minutos (lento) devido a menor solubilidade do TEOS nesse período de tempo; de 40 até 70 minutos (rápido), devido a maior solubilidade promovida pela geração de etanol como subproduto na solução e; de 70 até 120 minutos (lento) devido ao esgotamento de grupos alcóxi e pela polimeração das moléculas de silano;

Comprovou-se que há possibilidade de utilização de soluções com baixo teor alcoólico para a hidrólise do TEOS sem prejuízos à solução, mostrando-se como alternativa aos processos que utilizam alto consumo de solventes orgânicos.

\section{AGRADECIMENTOS}

Os autores gostariam de agradecer à CAPES (Ministério da Educação) no projeto 99999.000243/2015-09, ao CNPq e a FAPESC pelo apoio financeiro ao laboratório NanoTec-UFSC.

\section{BIBLIOGRAFIA}

[1] GUGLIELMI, M., CARTURAN, G., "Precursors for sol-gel preparations", Journal of Non-Crystalline Solids, v. 100, n. 1-3, pp. 16-30, March 1988.

[2] YOUNG, S.K., Overview of Sol-Gel Science and Technology, In: Report ARL-TR-2650, Army Research Laboratory, Aberdeen, MD., 2002.

[3] MACKENZIE, J.D., “Applications of the sol-gel process”, Journal of Non-Crystalline Solids, v. 100, n. 1-3, pp. 162-168, March 1988.

[4] LENZA, R.F.S., VASCONCELOS, W.L., "Preparation of silica by sol-gel method using formamide", Materials Research, v. 4, n. 3, pp. 189-194, July, 2001.

[5] SANTOS M.C.G., FREIRE, C.M.A., "Avaliação da resistência à corrosão de eletrodepósitos de liga ZnFe sobre aço tratado com filmes contendo sais de terras raras e silanos BTSE, BTESPTS", Matéria (Rio Janeiro), v. 18, n. 4, pp. 1576-1586, Maio, 2013.

[6] SANTOS, M.C.G., CÉLIA, M.A.F., “Avaliação por EIE do filme contendo o silano y-UPS como revestimento de passivação para as ligas $\mathrm{ZnFe}$ e ZnCo eletrodepositadas”, Matéria (Rio Janeiro), v. 18, n. 4, pp. 1459-1466, Setembro, 2013.

[7] VENKATESWARA RAO, A., BHAGAT, S.D., "Synthesis and physical properties of TEOS-based silica aerogels prepared by two step (acid-base) sol-gel process", Solid State Sciences, v. 6, n. 9, pp. 945-952, April 2004.

[8] BRINKER, C.J., SCHERER, G.W., Sol-Gel Science: The physics and chemistry of sol-gel processing, 1 ed., Academic Press. Inc., San Diego, 1990.

[9] TÉLlEZ, L., RUBIO, F., PEÑA-ALONSO, R., RUBIO, J., "Seguimiento por espectroscopia infrarroja (FT-IR) de la copolimerización de TEOS (tetraetilortosilicato) y PDMS (polidimetilsiloxano) en presencia de tbt (tetrabutiltitanio)", Boletin de la Sociedad Espanola de Ceramica y Vidrio, v. 43, n. 5, pp. 883-890, 2004.

[10] CIHLÁř́, J., "Hydrolysis and polycondensation of ethyl silicates. 1. Effect of pH and catalyst on the hydrolysis and polycondensation of tetraethoxysilane (TEOS)", Colloids and Surfaces A: Physicochemical and Engineering Aspects, v. 70, n. 3, pp. 239-251, 1993. 
[11] BRINKER, C.J., "Hydrolysis and condensation of silicates: Effects on structure", Journal of NonCrystalline Solids, v. 100, n. 1-3, pp. 31-50, 1988.

[12] VAN OOIJ, W.J., ZHU, D., STACY, M., et al., "Corrosion Protection Properties of Organofunctional Silanes - An Overview”, Tsinghua Science and Technology, v. 10, n. 6, pp. 639-664, Dec. 2005.

[13] PLUEDDEMANN, E.P., Silane Coupling Agents, 2 ed., Boston, Springer US, 1991.

[14] MASMOUDI, M., RAHAL, C., ABDELMOULEH, M., et al., "Hydrolysis process of $\gamma$-APS and characterization of silane film formed on copper in different conditions", Applied Surface Science, v. 286, pp. 71-77, 2013.

[15] DONATTI, D., IBAÑEZ RUIZ, A., VOLLET, D., "From sol to aerogel: a study of the nanostructural characteristics of TEOS derived sonogels", Journal of Non-Crystalline Solids, v. 292, n. 1-3, pp 44-49, 2001.

[16] CAPIOTTO, N., Uso de silano BSTE como protetivo contra a corrosão de laminados de aço carbono, Dissertação de M.Sc, Escola Politécnica da Universidade de São Paulo, São Paulo, SP, Brasil, 2006.

[17] RUBIO, F., RUBIO, J., OTEO, J.L., “A FT-IR Study of the Hydrolysis of Tetraethylorthosilicate (TEOS)", Spectroscopy Letters, v. 31, n. 1, pp. 199-219, 1998.

[18] AL-OWEINI, R., EL-RASSY, H., "Synthesis and characterization by FTIR spectroscopy of silica aerogels prepared using several $\mathrm{Si}(\mathrm{OR}) 4$ and $\mathrm{R}^{\prime}$ ' $\mathrm{Si}\left(\mathrm{OR}^{\prime}\right) 3$ precursors", Journal of Molecular Structure, v. 919, n. 1-3, pp. 140-145, 2009.

[19] LAZGHAB, M., SALEH, K., GUIGON, P., "Functionalization of porous silica powders in a fluidisedbed reactor with glycidoxypropyltrimethoxysilane (GPTMS) and aminopropyltriethoxysilane (APTES)", Chemical Engineering Research and Design, v. 88, n. 5-6, pp 686-692, 2010.

[20] KUNST, S.R., CARDOSO, H.R.P., OLIVEIRA, C.T., et al., "Corrosion resistance of siloxanepoly(methyl methacrylate) hybrid films modified with acetic acid on tin plate substrates: Influence of tetraethoxysilane addition", Applied Surface Science, v. 298, pp. 1-11, 2014.

[21] SILVERSTEIN, R., BLASSLER, G.C., TERENCE, C.M., Spectrometric identification of organic compounds, 5 ed., New York, NY, John Wiley \& sons, Inc., 1991.

[22] GIZDAVIC-NIKOLAIDIS, M.R., EDMONDS, N.R., BOLT, C.J., et al., "Structure and properties of GPTMS/DETA and GPTMS/EDA hybrid polymers", Current Applied Physics, v. 8, n. 3-4, pp 300-303, 2008.

[23] KHRAMOV, A., BALBYSHEV, V., VOEVODIN, N., et al., "Nanostructured sol-gel derived conversion coatings based on epoxy- and amino-silanes", Progress in Organic Coatings, v. 47, n. 3-4, pp. 207-213, 2013.

[24] TORRY, S.A., CAMPBELL, A.V., CUNLIFFE, D.A., "Kinetic analysis of organosilane hydrolysis and condensation”, International Journal of Adhesion and Adhesives, v. 26, pp. 40-49, 2006.

[25] CUNLIFFE, A.V., EVANS, S., TOD, D.A., et al., "Optimum preparation of silanes for steel pretreatment”, International Journal of Adhesion and Adhesives, v. 21, n. 4, pp. 287-296, 2001. 